Rahmenbedingungen für umweltbezogenes organisationales Lernen

\section{Impulse durch reflexives Recht}

\section{Eine nachhaltige Entwicklung erfordert gesellschaftliche Lernprozesse. Dazu be- darf es jedoch never Steuerungsmechanismen und Kontextbedingungen. Chancen hierfür bietet reflexives Umweltrecht, das stärker auf Selbst- regulierung setzt. Beispiele wie das europäische Öko-Audit-System zeigen, dass hierdurch Unternehmenslernen gefördert werden kann.}

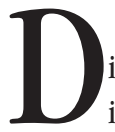

Von Martin Müller

ie bislang vorherrschende Umweltpolitik ist zielfixiert und fordert die Anpassung an einen Status quo. Auf gesellschaftlicher Ebene stellt Nachhaltigkeit diese vor die Herausforderung, die erforderlichen Such- und Lernprozesse für technische und organisatorische Innovationen anzustoßen. Dies erfordert einen Paradigmenwechsel hin zu einer ergebnisoffeneren Gestaltung der Umweltpolitik. Als Antwort auf diese Herausforderung hat sich das so genannte reflexive Recht entwickelt, welches auf regulierter Autonomie beruht. Es führt zu abstrakten prozeduralen Programmen, die sich auf die Meta-Ebene der Regulierung von Prozessen, Organisationsstrukturen, auf die Verteilung und Neudefinition von Steuerungsrechten und von Entscheidungskompetenzen konzentrieren. Das heißt, dass reflexives Recht aktiv selbst-regulierende, ,lernende“ Sozialsysteme fördert (1). Im Folgenden werden die Wirkungen auf umweltbezogenes Lernen in Unternehmen diskutiert, die von diesem Mechanismus ausgehen können.

Bezogen auf den Umweltkontext soll damit die interne selbstkritische Reflexion über die Umweltschutzleistungen innerhalb von Unternehmen gefördert werden. Der Regelungsmechanismus zielt weiterhin darauf ab, Prozesse in Gang zu setzen, welche ein reflexives Denken und Lernen über Umweltaspekte unterstïtzen. Die Rolle des Staates besteht dabei darin, die Wirtschaftssubjekte internen und externen Informations-, Lern- und Selbstkontrollprozessen auszusetzen. Das reflexive Umweltrecht strukturiert den Raum von Handlungsmöglichkeiten vor, indem es Organisationsstrukturen, Steuerungsrechte oder Entscheidungskompetenzen reguliert. Erste Ansätze eines reflexiven Rechts finden sich bei der obligatorischen Selbstkontrolle durch Betriebsbeauftragte im Immissionsschutz-, Abfall-, Wasser- und Strahlenschutz sowie in der europäischen Öko-Audit-Verordnung (EMAS) (2). Weitere Ansätze sind im Entwurf des vereinheitlichten
Umweltgesetzbuches zu finden, in dem eine Ausweitung der Mitteilungspflichten zur Betriebsorganisation festgeschrieben wurde.

\section{Stakeholder und öffentliches Wissen stärken}

Ein weiterer wichtiger Ansatzpunkt wäre eine Stärkung der Handlungsmacht von Nichtregierungsorganisationen und Stakeholdergruppen Ein strukturierter Dialog mit Stakeholdern, beispielsweise über eine Umweltberichterstattung vergegenwärtigt Problemstellungen und kann Auslöser für Lernvorgänge sein. Lernen ist demnach Ergebnis des misfit zwischen Organisationsrealität und Umweltanforderungen (3). Dadurch Unternehmen in Frage gestellt, wodurch es zu Lernprozessen kommen kann. Die Wirksamkeit dieses Mechanismus ist jedoch davon abhängig, ob und inwieweit die Umweltschutzleistungen von Unternehmen durch die Anspruchsgruppen beurchende Normsetzung erforderlich, die auch die Transparenz von Stoff- und Energieströmen einfordert. Umweltberichte in denen nicht nur über die Organisation des Umweltschutzes sondern auch über die Stoff- und Energieströme zu berichten wäre, setzten entsprechende Strukturen voraus, die solche Flüsse erfassen und bewerten Durch eine weitergehende Standardisierung einer Umweltberichterstattung lässt sich die Vergleichbarkeit verbessern, insofern besteht hier die Möglichkeit, im Sinne eines Benchmarking Lernprozesse zu erreichen.

Eine weitere Möglichkeit, organisationales Lernen durch geeignete Rahmenbedingungen zu fördern, ist eine strukturierte Sammlung und Veröffentlichung ökologischer Informationen bei einer zentralen Stelle, die auch für die Kommunikation dieser Informationen verantwortlich wäre. Ein derart institutionalisierter Kommunikationsprozess ermöglicht einen Zuwachs an öffentlichem werden die grundlegenden Handlungsmuster der teilt werden können. Hierfür ist eine entspre-
Wissen, da die Informationen allen Akteuren zugänglich sind. Erfahrungen, welche bislang nur innerhalb der Organisation gemacht wurden, sind damit im Sinne eines centers of expertise (4) tatsächlich für einen Diskurs verfügbar. Die Einrichtung einer solchen Stelle, die Weitergabe der Daten und Informationen durch die Unternehmen und die Aufbereitung und Kommunikation der Daten durch diese Stelle sollten Gegenstandsbereich einer reflexiven Normsetzung sein.

\section{Anforderungen an die Unternehmen}

$\mathrm{Zu}$ beachten ist allerdings, dass reflexives Recht darauf abzielt, interne Betriebsabläufe und Entscheidungen im Lichte von Umweltschutzaspekten kritisch zu hinterfragen. Damit sollen innerbetriebliche Lernprozesse angestoßen werden. Reflexives Recht muss, um Lernwirkungen zu ermöglichen, bis zu einem gewissen Grad unbestimmt bleiben. Es ist auf ein Verfahren ausgerichtet und nicht wie klassisches Ordnungsrecht auf materielle Details, welche dann wieder einen Status quo festschreiben würden. Daher ist es erforderlich, dass die Unternehmen ebenfalls Strukturen schaffen, um an die externen Vorgaben anzuschließen. Erst wenn Makroebene und Mesoebene aufeinander abgestimmt sind, kann es beim einzelnen Akteur zu Lernerfolgen kommen. Dies setzt allerdings entweder die Bereitschaft der Unternehmen voraus, umweltbezogene Lernwirkungen zu realisieren, oder eine Verknüpfung des reflexiven Rechts mit Anreizwirkungen, sodass entsprechende Strukturen im Unternehmen aufgebaut werden. Im Rahmen des EMAS-Prozesses werden seit längerem - leider mit wenig Erfolg - verschiedene Anreizmechanismen diskutiert. Organisationales Wissen ist gespeichert in anonymisierten Regelsystemen wie Leitlinien, Organisationsanweisungen, Prozessbeschreibungen, Routinen, Traditionen und der spezifischen Kultur einer Organisation. Organisationales Lernen vollzieht sich im Kontext dieser Strukturen, will diese aber auch ständig verändern. Dies bedeutet, dass umweltbezogenes organisationales Lernen auf der Unternehmensebene eine entsprechende Verankerung ökologischer Aspekte in den betrieblichen Strukturen, Prozessen und Abläufen voraussetzt. Hierfür bietet sich der Aufbau eines Umweltmanagementsystems an.

Grundlegend ist das Aufstellen von Umweltleitlinien für das Unternehmen. Die Formulierung solcher Umweltleitlinien für die Organisation kann als Lernprozess interpretiert werden, da ei- 
ne Reflexion über die eigenen umweltbezogenen Sollzustände - gerade in kleineren und mittleren Unternehmen - meist noch nicht stattgefunden hat. Bei Umweltleitlinien handelt es sich gewissermaßen um die offizielle umweltbezogene Handlungstheorie der Organisation.

\section{- Lernen durch Umweltmanage- mentsysteme}

Ein weiterer wichtiger Aspekt, der an die betriebliche Umweltpolitik anschließen muss, ist die Festlegung von umweltbezogenen Zielsetzungen und Einzelzielen, die dann im Rahmen eines Umweltmanagements abgearbeitet werden. Hier ist der Ort, um an reflexive Normsetzungen des Staates anzuknüpfen, indem festgelegt wird, wie Stoff- und Energieströme zu erfassen sind und welche Informationen gesammelt und bewertet werden.

Damit das Wissen der Mitarbeiter, etwa über ökologische Bewertungsverfahren, der Organisation zugänglich ist, muss es kommuniziert werden. Strukturen und Freiräumen, welche Kommunikation ermöglichen, kommt daher bei der Implementierung eines Umweltmanagementsystems eine zentrale Rolle zu. Notwendig sind im Rahmen des Umweltmanagements auch organisatorische Voraussetzungen für permanente umweltbezogene Weiterbildungsangebote, ein experimentierfreudiges und veränderungsbereites Klima am Arbeitsplatz, weitgehende Selbstbestimmung und Gelegenheit zur kritischen Reflexion der organisatorischen Realität.

Erst die Reflexion der Prozesse und Abläufe eines Umweltmanagementsystems ermöglicht aber organisationales Lernen. Die im Umweltmanagement spezifizierten Anforderungen beziehen sich auf die Dokumentation und Beurteilung von Messungen oder sonstigen Erfassungsmethoden, mit denen Umweltauswirkungen ermittelt werden. Mit der Verwirklichung dieser Anforderung werden lediglich ökologische single-loop-learning Prozesse unterstiitzt, da es sich hierbei um Soll-IstAbweichungsanalysen innerhalb des Systems handelt. Erst eine darüber hinausgehende Prüfung, die auf die Reflexion der grundlegenden Handlungsmuster zielt und somit double-loop-learning in ihrem Fokus hat, ermöglicht weiterführende Lernerfolge. Dabei ist die Befragung der Mitarbeiter das entscheidende Instrument, um Lernen zu unterstïtzen, denn das Bewusstwerden über eigenes Denken und Handeln erfolgt im Dialog.

Der Gedankenaustausch wird aus sicherer Distanz beobachtet und reflektiert. „Aus der Vogelperspektive des Beobachters können Muster erkannt werden, kann ein deutero-Lernen und ein ,reframing" erst möglich werden“ (5). Deutero-Lernen bezeichnet dabei die dritte Stufe organisationalen Lernens. Hier wird die Verbesserung der Lernfähigkeit einer Organisation selbst zum Gegenstand des Lernprozesses. Es geht um die Frage, welche Muster in Lernprozessen angelegt sind und wie die Einsicht in diese Muster zukünftige Lernprozesse beeinflusst.

Es darf nicht die Frage nach festgeschriebenen Organisationsstrukturen im Vordergrund stehen, sondern die Frage, ob die bestehenden umweltbezogenen Strukturen und Abläufe und ihre Funktion von den Mitarbeitern verstanden und mitgetragen werden.

\section{Fazit}

Lernen in Organisationen vollzieht sich in institutionell verankerten Strukturen. Hierbei dürfen formale Regelungen eines reflexiven Rechts nicht als Gegensatz, sondern als Ergänzung gesehen werden. Erforderlich ist ein Zusammenspiel öffentlicher und privater (unternehmensinterner) Akteure bzw. öffentlicher und privater Systeme, um umweltbezogenes organisationales Lernen zu ermöglichen.

\section{Anmerkungen}

(1) Vgl. Teubner, G./ Willke, H.: Kontext und Autonomie: Gesellschaftliche Selbststeuerung durch reflexives Recht, EUI Working Paper Nr. 93, European University Institute, Florenz 1984.

(2) Vgl. Müller, M.: Mit reflexivem Recht zu lernendem Umweltmanagement, in: Zeitschrift für Umweltpolitik und Umweltrecht, Nr. 1/2002, S. 1-26.

(3) Vgl. Pawlowsky, P.: Betriebliche Qualifikationsstrategien und organisationales Lernen, in: Staehle, W. H./ Conrad, P. (Hrsg.): Managementforschung 2, Berlin, New York 1992, S. 177-237.

(4) Willke, H. : Dimensionen des Wissensmanagements Zum Zusammenhang von gesellschaftlicher und organisationaler Wissensbasierung, in: Schreyögg, G./ Conrad, P. (Hrsg.): Managementforschung 6, Berlin, New York 1996, S. 263-304.

(5) Pawlowsky a.a.0., S. 223.

\section{Der Autor}

Dr. Martin Müller ist wissenschaftlicher Mitarbeiter an der Carl von Ossietzky-Universität Oldenburg.

Kontakt: Uni Oldenburg, Institut für Betriebswirtschaftslehre I, Lehrstuhl für Produktion und Umwelt, Gebäude A5, 26111 Oldenburg.

Tel. 0441/ 798-4187, Fax -4193,

E-Mail: martin.mueller@uni-oldenburg.de

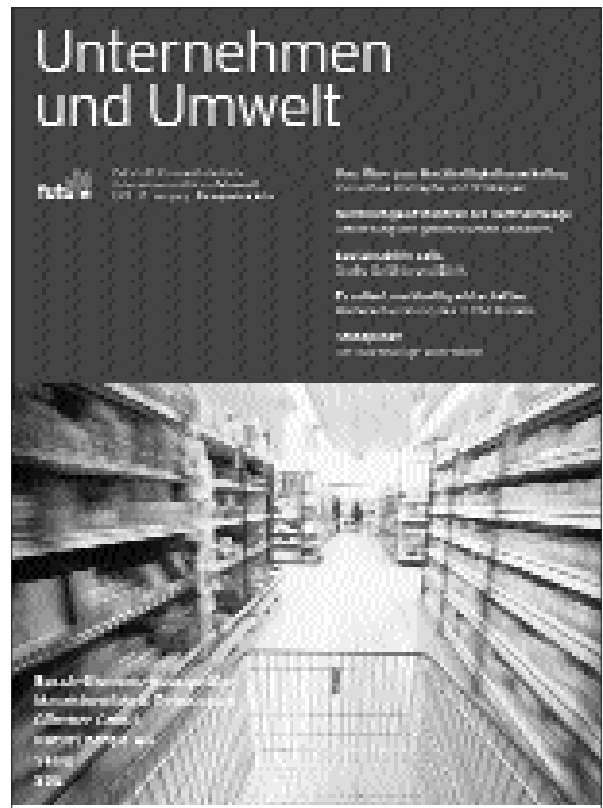

\section{Unternehmen und Umwelt}

.. ist die Zeitschrift für umweltorientierte Unternehmenspolitik von future e.V.

.. bietet Fachinformationen zum Umweltschutz und Best-Practice-Beispiele aus Unternehmen.

bietet viermal im Jahr Unternehmensbeispiele, Literatur- und Veranstaltungshinweise.

Mit einem Schwerpunktthema in jedem Heft. Zum Beispiel:

2/02: Ökomarketing

1/02: Nachhaltigkeit und Gewerbebau

4/01: Nachhaltigkeit im Handwerk

3/01: Klimaschutz

2/01: Nachhaltiges Investment

1/01: Grüne Bauwirtschaft

4/00: Kooperative Textilwirtschaft

3/00: Integrierte Produktpolitik

2100: Soft Facts im Umweltschutz

1/00: Ökologische Intelligenz

Bestellen Sie ihr kostenloses

Probe-Abo im Internet unter

w w w . f u t u re - e v. de

future e.V. Büro Bochum

Am Varenholt 123

44797 Bochum

Fon: $0234 / 9799513$

Fax: $0234 / 9799514$

bochum@future-ev.de

www.future-ev.de 
(c) 20I0 Authors; licensee IÖW and oekom verlag. This is an article distributed under the terms of the Creative Commons Attribution Non-Commercial No Derivates License (http://creativecommons.org/licenses/by-nc-nd/3.o/), which permits unrestricted use, distribution, and reproduction in any medium, provided the original work is properly cited. 